\title{
Global miRNA expression profiling of domestic cat livers following acute Toxoplasma gondii infection
}

\author{
Wei Cong ${ }^{1,2,{ }^{*}}$, Xiao-Xuan Zhang ${ }^{1, *}$, Jun-Jun $\mathrm{He}^{1}$, Fa-Cai $\mathrm{Li}^{1}$, Hany M. Elsheikha ${ }^{3}$, \\ Xing-Quan Zhu ${ }^{1}$ \\ ${ }^{1}$ State Key Laboratory of Veterinary Etiological Biology, Lanzhou Veterinary Research Institute, Chinese Academy of \\ Agricultural Sciences, Lanzhou, Gansu Province 730046, PR China \\ ${ }^{2}$ College of Marine Science, Shandong University at Weihai, Weihai, Shandong Province 264209, PR China \\ ${ }^{3}$ Faculty of Medicine and Health Sciences, School of Veterinary Medicine and Science, University of Nottingham, Sutton \\ Bonington Campus, Loughborough, LE12 5RD, UK \\ *These authors have contributed equally to this work \\ Correspondence to: Hany M. Elsheikha, email: hany.elsheikha@nottingham.ac.uk \\ Xing-Quan Zhu, email: xingquanzhul@hotmail.com
}

Keywords: Toxoplasma gondii, domestic cat, liver, mircoRNA, RNA-seq

Received: December 20, $2016 \quad$ Accepted: February 08, $2017 \quad$ Published: March 10, 2017

Copyright: Cong et al. This is an open-access article distributed under the terms of the Creative Commons Attribution License (CC-BY), which permits unrestricted use, distribution, and reproduction in any medium, provided the original author and source are credited

\section{ABSTRACT}

Although microRNAs (miRNAs) play an important role in liver homeostasis, the extent to which they can be altered by Toxoplasma gondii infection is unknown. Here, we utilized small RNA sequencing and bioinformatic analyses to characterize miRNA expression profiles in the liver of domestic cats at 7 days after oral infection with T. gondii (Type II) strain. A total of 384 miRNAs were identified and 82 were differentially expressed, of which 33 were up-regulated and 49 down-regulated. Also, 5690 predicted host gene targets for the differentially expressed miRNAs were identified using the bioinformatic algorithm miRanda. Gene ontology analysis revealed that the predicted gene targets of the dysregulated miRNAs were significantly enriched in apoptosis. Kyoto Encyclopedia of Genes and Genomes analysis showed that the predicted gene targets were involved in several pathways, including acute myeloid leukemia, central carbon metabolism in cancer, choline metabolism in cancer, estrogen signaling pathway, fatty acid degradation, lysosome, nucleotide excision repair, progesterone-mediated oocyte maturation, and VEGF signaling pathway. The expression level of 6 upregulated miRNAs (mmu-miR-21a-5p, mmu-miR-20a-5p, mmu-miR-17-5p, mmu-miR-30e-3p, mmu-miR142a-3p, and mmu-miR-106b-3p) was confirmed by stem-loop quantitative reverse transcription PCR, which yielded results consistent with the sequencing data. These findings expand our understanding of the regulatory mechanisms of miRNAs underlying T. gondii pathogenesis and contribute new database information on cat miRNAs, opening a new perspective on the prevention and treatment of $T$. gondii infection.

\section{INTRODUCTION}

Toxoplasma gondii is a highly prevalent apicomplexan protozoan parasite, which can cause serious clinical illnesses in humans and animals [1]. It has been reported to chronically infect roughly one-third of the world's human population [2]. T. gondii acquired during pregnancy may cause damage to the fetus and reactivation of latent infection can cause life-threatening encephalitis in immune-compromised individuals [3]. This parasite has an indirect two-host lifecycle, which is composed of asexual reproductive phase in the intermediate host and sexual reproductive phase in the definitive host (members of the Felidae family). The enteroepithelial sexual cycle of $T$. gondii is completed within 3 to 10 days after ingestion of intermediate host tissue containing $T$. gondii cysts. T. gondii can also spread throughout the cat's body and affect many organs 
[4-7]. Hence, cats are unique in respect of their ability to accommodate both sexual and asexual reproductions of $T$. gondii, making cats a significant source of infection to humans and animals $[2,8]$.

Besides the adverse clinical consequences on humans and other intermediate hosts $T$. gondii can cause disseminated and fatal infection in cats [4-7, 9]. Although any organ in the cat's body can be affected, clinical cases related to hepatic and pulmonary damage are particularly important because they are associated with quicker mortality [6, 10-12]. Also, liver dysfunction, enlargement, icterus, cholangiohepatitis, vomiting, abdominal effusion, and ascites are complications that frequently occur in $T$. gondii-infected cats. Therefore, with the great need for the development of efficacious treatment interventions (due to the lack of a vaccine and limited efficacies of current therapeutics), it is important to identify the molecular mechanisms that underpin liver damage caused by $T$. gondii infection. However, information about the molecular pathways that regulate the interaction between $T$. gondii and hepatic tissues has been limited to a few studies [13, 14].

The feline genome already encodes roughly 3,182 microRNA (miRNA) homologues, which can regulate the expression of signalling cascades that perform key cellular functions, such as cell cycle regulation, proliferation, differentiation, apoptosis, and carcinogenesis. miRNAs constitute a group of endogenous non-coding small RNAs (18 to 25 nucleotides [nt] long) that regulate gene expression by binding to mRNA and inhibiting translation [15-18]. miRNAs play an important role in liver homeostasis, and aberrant expression of miRNAs has been associated with a variety of liver diseases, such as viral hepatitis, hepatocellular carcinoma and fatty liver disease [19]. Alterations of host miRNA expression have also been observed in some parasitic infections, such as Cryptosporidium parvum, Plasmodium falciparum and $T$. gondii (reviewed in [20]), underscoring the potential role of miRNAs in mediating the interaction between $T$. gondii and host cells. Despite the impact of $T$. gondii infection on hepatic function the mechanisms underlying the alterations of hepatic miRNAs expression following acute $T$. gondii infection remain poorly understood.

In this study, we hypothesized that $T$. gondii infection alters the expression of hepatic miRNAs and that differentially expressed miRNAs mediate the interaction between $T$. gondii and cat's liver. Here we use genomewide, small RNA sequencing to characterize the global miRNA transcriptional response of feline liver to infection with $T$. gondii (Type II) PRU strain. Our study provides a full picture of the hepatic miRNA repertoire during acute $T$. gondii infection in domestic cats, including novel miRNAs, involved in host cell cycle, apoptosis and anti- $T$. gondii defense.

\section{RESULTS}

\section{Confirmation of $T$. gondii infection in the cat livers}

Under the conditions we used positive PCR results were obtained, providing laboratory confirmation of T. gondii infection in the livers of infected cats. RFLP analysis of the positive PCR amplicons of $T$. gondii B1 gene revealed a restriction fragment pattern characteristic to T. gondii genotype II. The livers of control cats and negative PCR control yielded negative PCR results.

\section{Analysis of miRNA expression}

miRNA libraries of livers from two T. gondii-infected or two control cat groups were successfully sequenced and sequencing data is summarized in Table 1 and Table 2. Length distributions of clean reads in the libraries were between 20-24 nt (Figure 1A-1D). A very high intra-group correlation was detected between the two miRNA libraries of $T$. gondii-infected liver samples $\left(\mathrm{R}^{2}=0.989\right)($ Figure $2 \mathrm{~A}$ ) and the two miRNA libraries of uninfected liver samples $\left(\mathrm{R}^{2}\right.$ $=0.99)$ (Figure 2B). The known and novel mature miRNAs in $T$. gondii-infected and control groups were summarized in Table 3 and Table 4. Finally, through comparing T. gondiiinfected and uninfected sRNA libraries, 82 differentially expressed miRNAs were identified, including 33 upregulated and 49 down-regulated miRNAs (Table 5).

\section{Pathway analysis of miRNA targets}

A total of 5690 predicted host targets were identified (Supplementary Table 2). Based on the predicted targets of the differentially expressed miRNAs GO enrichment analysis was performed in order to identify the biological processes, molecular functions and cellular components. The enriched GO terms of the biological processes, molecular functions and cellular components are shown in Figure 3, respectively. KEGG enrichment analysis showed that target genes were related to multiple pathways, including nucleotide excision repair, lysosome, vascular endothelial growth factor (VEGF) signaling, estrogen signaling, acute myeloid leukemia, central carbon metabolism in cancer, choline metabolism in cancer, fatty acid degradation, progesterone-mediated oocyte maturation, and renal cell carcinoma. The top 20 KEGG enrichment pathways are shown in Figure 4.

\section{miRNA expression validation by $q$ RT-PCR}

Six miRNAs, including mmu-miR-21a-5p, mmumiR-20a-5p, mmu-miR-17-5p, mmu-miR-30e-3p, mmumiR-142a-3p, and mmu-miR-106b-3p, were selected for 
Table 1: Summary of small RNA sequencing data obtained in the present study

\begin{tabular}{lcccccc}
\hline Library type & Reads & Bases & Error rate & Q20 & Q30 & GC content \\
\hline Infected liver Group 1 & 10665548 & $0.533 \mathrm{G}$ & $0.01 \%$ & $96.21 \%$ & $92.13 \%$ & $49.65 \%$ \\
Infected liver Group 2 & 12569494 & $0.628 \mathrm{G}$ & $0.01 \%$ & $96.16 \%$ & $92.13 \%$ & $49.98 \%$ \\
Uninfected liver Group 1 & 11727706 & $0.586 \mathrm{G}$ & $0.01 \%$ & $96.24 \%$ & $92.33 \%$ & $49.56 \%$ \\
Uninfected liver Group 2 & 10273236 & $0.514 \mathrm{G}$ & $0.01 \%$ & $96.44 \%$ & $92.60 \%$ & $49.72 \%$ \\
\hline
\end{tabular}

Table 2: Summary of the standard bioinformatic quality check and cleaning of small RNAs

\begin{tabular}{|c|c|c|c|c|}
\hline \multirow[b]{2}{*}{ Library } & \multicolumn{2}{|c|}{ Infected groups } & \multicolumn{2}{|c|}{ Uninfected groups } \\
\hline & $\begin{array}{l}\text { Infected liver } \\
\text { Group } 1\end{array}$ & $\begin{array}{l}\text { Infected liver } \\
\text { Group } 2\end{array}$ & $\begin{array}{c}\text { Uninfected liver } \\
\text { Group } 1\end{array}$ & $\begin{array}{c}\text { Uninfected liver } \\
\text { Group } 2\end{array}$ \\
\hline Total reads & $10665548(100.00 \%)$ & $12569494(100.00 \%)$ & $11727706(100.00 \%)$ & $10273236(100.00 \%)$ \\
\hline $\mathrm{N} \%>10 \%$ & $531(0.00 \%)$ & $636(0.01 \%)$ & $606(0.01 \%)$ & $540(0.01 \%)$ \\
\hline Low quality & $40551(0.38 \%)$ & $47641(0.38 \%)$ & $42076(0.36 \%)$ & $32984(0.32 \%)$ \\
\hline 5_adapter_contamine & $1247(0.01 \%)$ & $1565(0.01 \%)$ & $1026(0.01 \%)$ & $915(0.01 \%)$ \\
\hline $\begin{array}{l}\text { 3_adapter_null or } \\
\text { insert_null }\end{array}$ & $174157(1.63 \%)$ & $176650(1.41 \%)$ & $223708(1.91 \%)$ & $195007(1.90 \%)$ \\
\hline With ployA/T/G/C & $10772(0.10 \%)$ & $12621(0.10 \%)$ & $7991(0.07 \%)$ & $6939(0.07 \%)$ \\
\hline Clean reads & $10438290(97.87 \%)$ & $12330381(98.10 \%)$ & $11452299(97.65 \%)$ & $10036851(97.70 \%)$ \\
\hline
\end{tabular}

confirmation by real time PCR to verify the expression levels of the differentially expressed miRNAs using miRNA specific primers (Supplementary Table 1). The results confirmed that these selected miRNAs were differentially expressed between infected livers and uninfected livers and were consistent with the results obtained by RNAsequencing analysis (Supplementary Figure 1). Data set from the RNA-sequencing experiment has been deposited in the GEO database under accession number PRJNA356106.

\section{DISCUSSION}

Previous studies showed that $T$. gondii infection can alter the expression of host miRNAs, indicating that miRNAs may be involved in the pathogenesis of $T$. gondii infection [21-24]. Our results have also shown that acute $T$. gondii infection alters the level of miRNAs in the liver of domestic cats. In the present study, we used small RNA sequencing to identify cellular miRNAs and signaling pathways involved in the response of cats to $T$. gondii infection. Specifically, we determined miRNAs that are differentially expressed by comparing sham-infected to T. gondii-infected cat livers at 7 days after infection. This analysis identified 82 differentially expressed microRNAs, of which 33 were increased and 49 decreased. By using qRT-PCR, the expression level of six up-regulated miRNAs (mmu-miR-21a-5p, mmu-miR-20a-5p, mmu-miR-17-5p, mmu-miR-30e-3p, mmu-miR-142a-3p, and mmu-miR- 106b-3p) were consistent with the results obtained by sequencing analysis. Knowledge of molecular changes in human liver during the acute phase of toxoplasmosis is lacking due to the mild and subtle nature of this infection especially in immune-component individuals $[1,2]$. Hence, these findings may serve as the basis for understanding the molecular mechanisms associated with hepatic pathology during acute $T$. gondii infection.

Differentially expressed miRNAs were associated with signalling pathways involved mainly in cell cycling, apoptosis, oncogenesis, and host defense. Among the differentially expressed miRNAs, miR-21a-5p, miR17-5p, miR-223-3p, miR-27a-5p, miR-126, and miR486 were significantly upregulated in $T$. gondii-infected livers compared to controls. Of note, the level of miR$21 \mathrm{a}-5 \mathrm{p}$ expression was elevated in various cancer tissues, including rectal, gastric and lung tissues [25-27] and has been suggested to play a role in tumor biology [27]. The similarity between the expression of miR-21a-5p during $T$. gondii infection and various forms of cancers is interesting. One striking finding was the correlative link between upregulation of miR-17-92 in T. gondiiinfected human foreskin fibroblasts [28] and in human astrocyticglioma tissue [29]. The presence of RNA silencing machinery and small silencing RNAs in $T$. gondii genome [30] indicates that this parasite has the ability to use its own miRNAs to interrupt host cell functions in analogy to oncogenic viruses [31]. 
miR-17-5p, a key regulator of the G1/S phase cell cycle transition, was up-regulated in our study in agreement with others who reported overexpression of miR-17-5p in human and mouse spleen in response to $T$. gondii infection $[21,31]$. T. gondii can increase miR-17 92 and miR-106b 25 that play key roles in the regulation of mammalian cell cycle by influencing the functionally intertwined pathways of apoptosis and G1/S cell cycle progression [32]. miR-17-5p targets mouse Bcl2111, Zmat3, Aifm1, and Capn2 to increase host apoptotic process and targets mouse Ppp3r1 and Akt3 to promote cellular apoptosis process [21]. Also, miR-17-5p may function as both a tumor suppressor [33] and as an oncogenic activator [34] by targeting both anti- and proproliferative genes and by competing with each other in different cellular contexts [35]. The effect of $T$. gondii infection on the expression of miRNAs (miR-30c-1, miR125b-2, miR-23b-27b-24-1, and miR-17 92 cluster genes) that have anti-apoptotic activity has been reported [36]. Modulating these apoptosis-related miRNAs with mimics
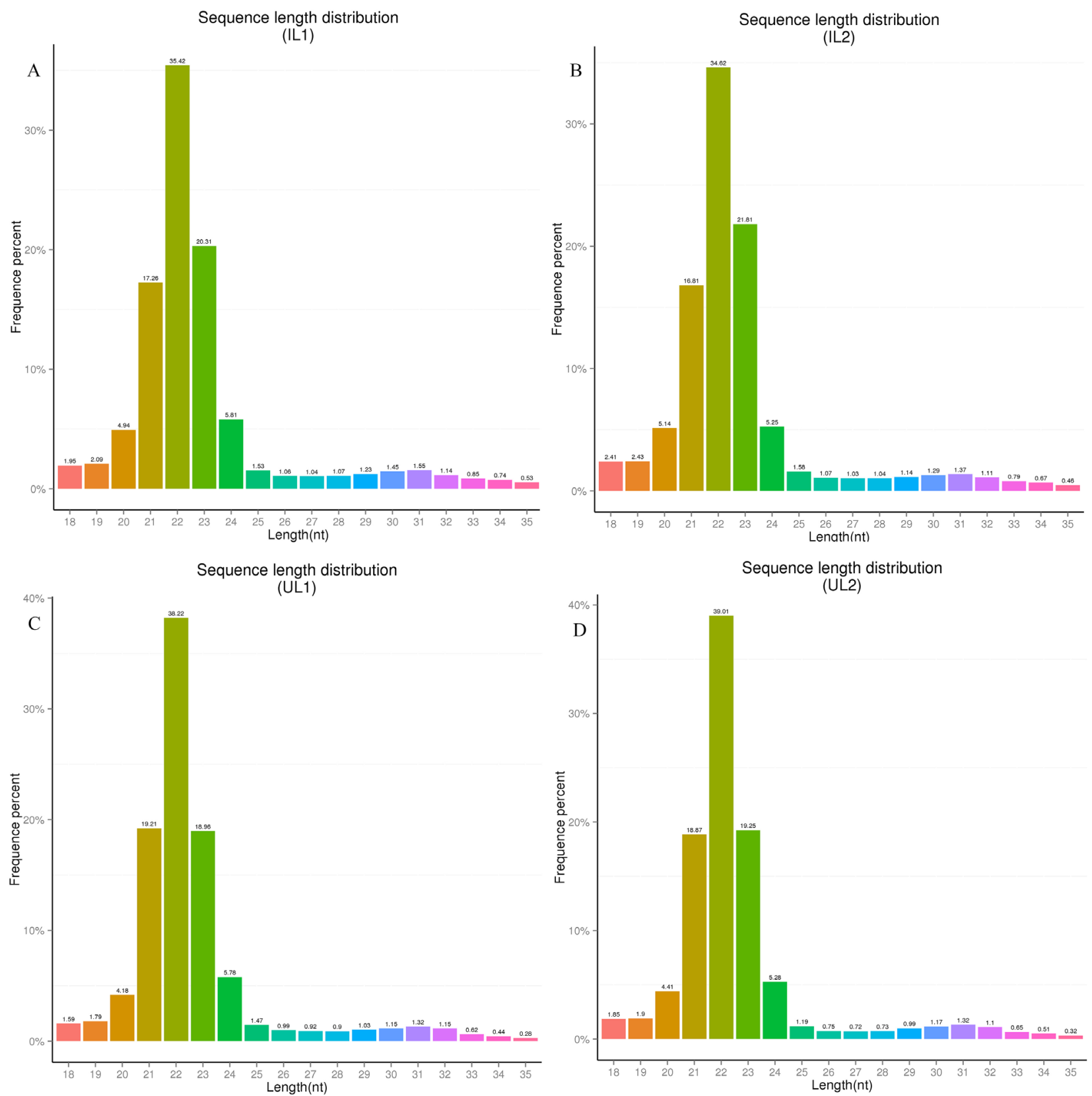

Figure 1: Length distribution of the small RNA expressions in the liver of domestic cats infected with Toxoplasma gondii compared to control cats. (A and B) Group 1 and group 2 of $T$. gondii-infected domestic cats; (C and D) Group 1 and group 2 of uninfected, control, domestic cats. Abbreviations: IL1, IL2, UL1, and UL2: infected liver 1, infected liver 2, uninfected liver 1, uninfected liver 2 , respectively. 
or inhibitors can validate their roles in the dysregulation of host cell apoptotic machinery during $T$. gondii infection.

The miR-223-3p has been implicated in the regulation of inflammatory response [37] and granulocyte production and function [38], and can function as a tumor suppresser in osteosarcoma by regulating the osteosarcoma cell cycle progression and proliferation [39]. The level of miR223-3p was significantly increased in infected samples, suggesting that $T$. gondii infection of feline liver stimulates the production of miR-223-3p, which plays a role in the activation of inflammatory response elicited in response to $T$. gondii infection. This is concordant with a previous study showing that miRNAs, such as miR-146a and miR155 , known to activate immune and inflammatory responses can influence host response to T. gondii infection [40-43]. Also, the upregulation of miR-27a-5p (a regulator of lipid metabolism-related genes) and miR-21-5p in the infected liver samples suggests that both miR-27a-5p and miR-21-5p play a role in host response to infection. This assumption is supported by the association between inhibition of miR-21 and increased Cryptosporidium parvum burden [29].

miR-126 is associated with tumorigenesis and has recently been found to modulate the survival and function of Plasmacytoid dendritic cells ( $\mathrm{pDCs}$ ) via positive regulation of the vascular endothelial growth factor (VEGF) signaling pathway [44]. miR-126 upregulation may activate Toll-like receptor (TLR)/MyD88 signalling in pDCs to secrete large amounts of type I interferons (IFNs), which is essential for host resistance to $T$. gondii infection. Also, the biological significance of $T$. gondii-induced upregulation of miR-486 may lie in its ability to augment the host defense mechanisms.
miR-486 has been shown to activate nuclear factor (NF)- $\kappa$ B signaling pathway [45], which leads to the production of proinflammatory cytokines, thereby providing a protection against $T$. gondii infection. Of note, both miR-486 and the NF- $\kappa \mathrm{B}$ signaling pathway have oncogenic roles in human cancers, such as glioma progression [45].

The let-7 family is a key regulator of the innate immune response. The level of let-7i during protozoal infection with $C$. parvum infection was found reduced together with increase of TLR4 in biliary epithelial cells, contributing to cholangiocyte's defense responses [46]. In line with this study our results revealed significant downregulation of mmu-let-7f-5p, mmu-let-7i-5p, mmulet-7a-5p, mmu-let-7g-5p, mmu-let-7e-5p, and mmu-let$7 \mathrm{c}-5 \mathrm{p}$ in $T$. gondii-infected cat's livers compared with controls. Finally, the fact that miRNAs are host-, tissue-, and strain-specific [47] explains why the expression of some miRNA (e.g. miR-712-3p, miR-511-5p and miR$217-5 \mathrm{p}$ ) that are dysregulated during $T$. gondii infection [48] was not altered in our study.

In conclusion, these findings provided new insight regarding the ability of $T$. gondii to alter the expression of 82 microRNAs in cat liver. Our study revealed that through reprogramming of hepatic miRNAs expression $T$. gondii influences the cellular microenvironment and host anti- $T$. gondii response, which are likely to play roles in the parasite pathogenesis. GO analysis revealed that the predicted targets of the differentially expressed miRNAs were involved in the regulation of cell cycle and most of the identified KEGG pathways were related to cancer. Considering the immunoregulatory effects of miRNAs and their ability to
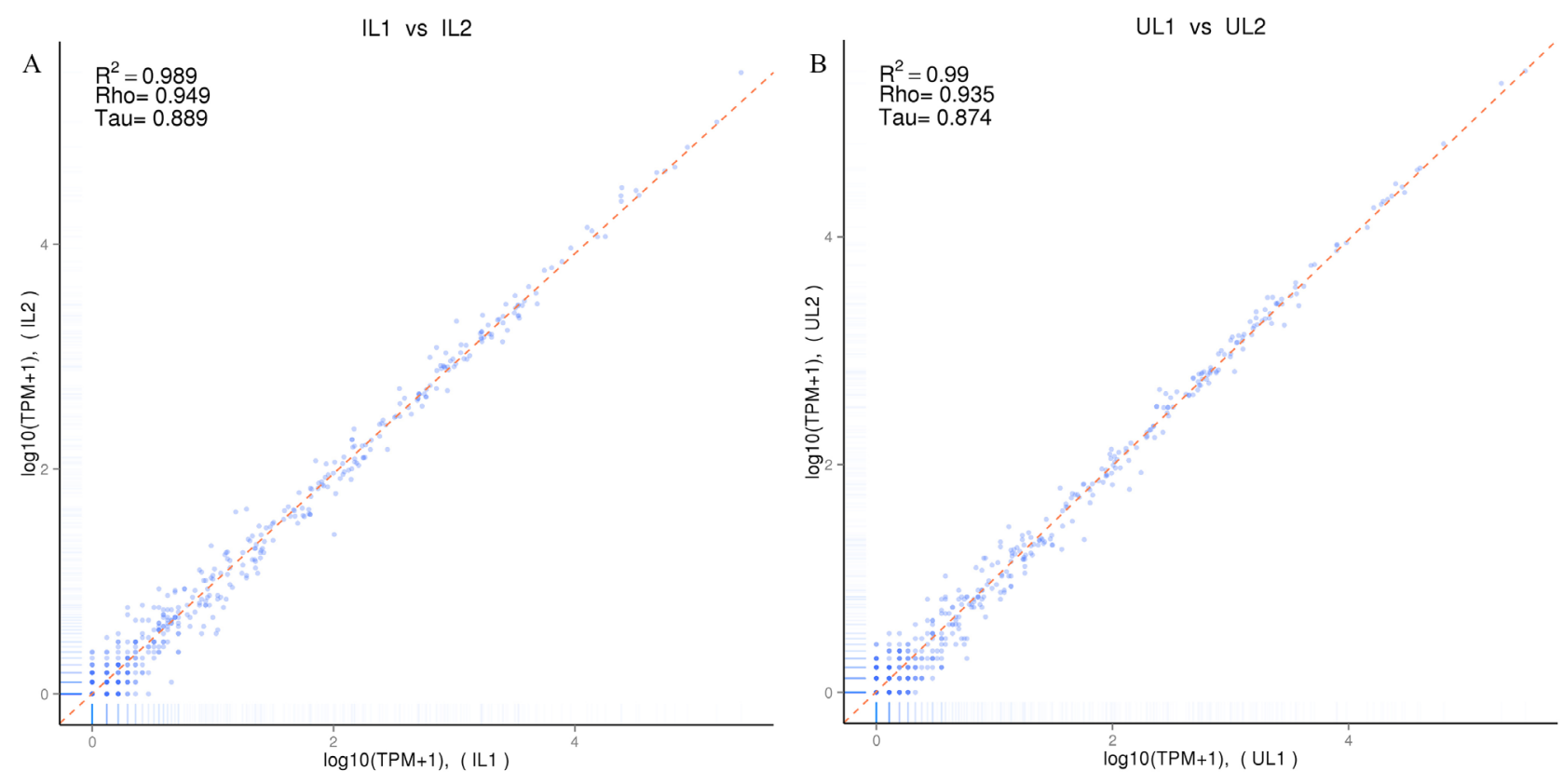

Figure 2: Correlation analysis of the small RNA expressions in the livers of $T$. gondii-infected and uninfected cats. (A) Correlation analysis of small RNA expression levels between T. gondii-infected cats of group 1 and group 2 (IL1 vs IL2); (B) Correlation analysis of small RNA expression levels between uninfected cats of group 1 and group 2 (U11 vs UL2). 
Table 3: Known miRNA mapping

\begin{tabular}{lccccc}
\hline \multirow{2}{*}{ Criteria } & \multicolumn{2}{c}{ Infected groups } & & \multicolumn{2}{c}{ Uninfected groups } \\
\cline { 2 - 3 } \cline { 5 - 6 } & $\begin{array}{c}\text { Infected liver } \\
\text { Group 1 }\end{array}$ & $\begin{array}{c}\text { Infected liver } \\
\text { Group 2 }\end{array}$ & & $\begin{array}{c}\text { Uninfected liver } \\
\text { Group 1 }\end{array}$ & $\begin{array}{c}\text { Uninfected liver } \\
\text { Group 2 }\end{array}$ \\
\hline Mapped mature & 358 & 360 & & 348 & 356 \\
Mapped hairpin & 273 & 274 & & 266 & 268 \\
Mapped uniq sRNA & 2531 & 2604 & & 2411 & 2409 \\
Mapped total sRNA & 3086174 & 3665542 & & 3484600 & 3037510 \\
\hline
\end{tabular}

Table 4: Novel miRNA mapping

\begin{tabular}{lcccc}
\hline Types & $\begin{array}{c}\text { Infected liver } \\
\text { Group 1 }\end{array}$ & $\begin{array}{c}\text { Infected liver } \\
\text { Group 2 }\end{array}$ & $\begin{array}{c}\text { Uninfected liver } \\
\text { Group 1 }\end{array}$ & $\begin{array}{c}\text { Uninfected liver } \\
\text { Group 2 }\end{array}$ \\
\hline Novel mature & 69 & 76 & 75 & 73 \\
Novel star & 10 & 14 & 12 & 10 \\
Novel hairpin & 72 & 78 & 80 & 77 \\
Mapped uniq sRNA & 186 & 187 & 161 & 161 \\
Mapped total sRNA & 12381 & 12103 & 13702 & 7981 \\
\hline
\end{tabular}

Table 5: Differentially expressed miRNAs

\begin{tabular}{lcccc}
\hline Type & miRNA & Fold change & $\boldsymbol{P}$-value & $\boldsymbol{P}$-adjustment \\
\hline Up-regulated & mmu-miR-21a-5p & 2.564 & $1.32 \mathrm{E}-17$ & $3.15 \mathrm{E}-15$ \\
mmu-miR-20a-5p & 1.610 & $3.26 \mathrm{E}-09$ & $3.88 \mathrm{E}-07$ \\
mmu-miR-339-5p & 1.839 & $5.42 \mathrm{E}-06$ & 0.0002 \\
mmu-miR-101a-3p & 1.855 & $8.22 \mathrm{E}-06$ & 0.0002 \\
mmu-miR-320-3p & 1.507 & $9.27 \mathrm{E}-06$ & 0.0002 \\
mmu-miR-195a-5p & 1.805 & $1.33 \mathrm{E}-05$ & 0.0003 \\
mmu-miR-126a-3p & 1.486 & $2.05 \mathrm{E}-05$ & 0.0003 \\
mmu-miR-23a-3p & 1.585 & $2.13 \mathrm{E}-05$ & 0.0003 \\
mmu-miR-140-3p & 1.701 & $2.45 \mathrm{E}-05$ & 0.0003 \\
mmu-miR-28a-5p & 1.500 & 0.0002 & 0.0018 \\
mmu-miR-223-3p & 1.917 & 0.0002 & 0.0023 \\
mmu-miR-30f & 1.504 & 0.0002 & 0.0023 \\
mmu-miR-126a-5p & 1.437 & 0.0003 & 0.0025 \\
mmu-miR-126b-3p & 1.435 & 0.0003 & 0.0025 \\
mmu-miR-17-5p & 1.436 & 0.0005 & 0.0036
\end{tabular}

(Continued) 


\begin{tabular}{|c|c|c|c|c|}
\hline Type & miRNA & Fold change & $P$-value & $P$-adjustment \\
\hline & mmu-miR-19b-3p & 1.485 & 0.0007 & 0.0048 \\
\hline & mmu-miR-27a-5p & 1.741 & 0.0011 & 0.0076 \\
\hline & mmu-miR-486b-3p & 1.593 & 0.0011 & 0.0076 \\
\hline & mmu-miR-151-3p & 1.338 & 0.0017 & 0.0093 \\
\hline & mmu-miR-27a-3p & 1.389 & 0.0017 & 0.0093 \\
\hline & mmu-miR-486a-3p & 1.571 & 0.0017 & 0.0093 \\
\hline & mmu-miR-99b-5p & 1.359 & 0.0021 & 0.0106 \\
\hline & novel_1 & 1.998 & 0.0030 & 0.0134 \\
\hline & mmu-miR-3074-5p & 1.315 & 0.0049 & 0.0202 \\
\hline & mmu-miR-30e-3p & 1.325 & 0.0052 & 0.0211 \\
\hline & mmu-miR-24-3p & 1.306 & 0.0055 & 0.0212 \\
\hline & mmu-miR-101c & 1.052 & 0.0069 & 0.0252 \\
\hline & mmu-miR-378c & 1.528 & 0.0069 & 0.0252 \\
\hline & mmu-miR-486a-5p & 1.494 & 0.0075 & 0.0271 \\
\hline & mmu-miR-361-3p & 1.379 & 0.0101 & 0.0342 \\
\hline & mmu-miR-106b-3p & 1.255 & 0.0153 & 0.0451 \\
\hline & mmu-miR-142a-3p & 1.690 & 0.0152 & 0.0451 \\
\hline & mmu-miR-142b & 1.690 & 0.0152 & 0.0451 \\
\hline \multirow[t]{15}{*}{ Down-regulated } & mmu-let-7f-5p & -1.546 & $1.73 \mathrm{E}-07$ & $1.38 \mathrm{E}-05$ \\
\hline & mmu-let-7i-5p & -1.705 & $1.37 \mathrm{E}-06$ & $6.52 \mathrm{E}-05$ \\
\hline & mmu-miR-365-3p & -1.532 & $1.24 \mathrm{E}-06$ & $6.52 \mathrm{E}-05$ \\
\hline & mmu-miR-148a-3p & -1.662 & $6.17 \mathrm{E}-06$ & 0.0002 \\
\hline & mmu-miR-381-3p & -2.264 & 4.87E-06 & 0.0002 \\
\hline & mmu-miR-370-3p & -2.876 & $8.54 \mathrm{E}-06$ & 0.0002 \\
\hline & mmu-miR-3071-5p & -2.007 & $1.62 \mathrm{E}-05$ & 0.0003 \\
\hline & mmu-miR-136-3p & -1.987 & $2.23 \mathrm{E}-05$ & 0.0003 \\
\hline & mmu-miR-30c-2-3p & -1.683 & $6.94 \mathrm{E}-05$ & 0.0009 \\
\hline & mmu-miR-128-3p & -1.487 & $7.45 \mathrm{E}-05$ & 0.0009 \\
\hline & mmu-miR-30d-5p & -1.358 & 0.0002 & 0.0018 \\
\hline & mmu-let-7a-5p & -1.426 & 0.0002 & 0.0023 \\
\hline & mmu-miR-340-5p & -1.570 & 0.0002 & 0.0023 \\
\hline & mmu-let-7g-5p & -1.349 & 0.0003 & 0.0023 \\
\hline & mmu-miR-493-5p & -2.565 & 0.0003 & 0.0023 \\
\hline
\end{tabular}




\begin{tabular}{|c|c|c|c|c|}
\hline Type & miRNA & Fold change & $P$-value & $P$-adjustment \\
\hline & mmu-miR-218-5p & -1.618 & 0.0003 & 0.0025 \\
\hline & mmu-miR-127-3p & -1.517 & 0.0003 & 0.0026 \\
\hline & mmu-let-7e-5p & -1.380 & 0.0006 & 0.0042 \\
\hline & mmu-miR-98-5p & -1.452 & 0.0012 & 0.0077 \\
\hline & mmu-miR-409-3p & -1.919 & 0.0013 & 0.0083 \\
\hline & mmu-let-7c-5p & -1.572 & 0.0015 & 0.0089 \\
\hline & mmu-miR-139-5p & -1.418 & 0.0015 & 0.0089 \\
\hline & mmu-miR-129b-3p & -1.526 & 0.0017 & 0.0093 \\
\hline & mmu-miR-129-5p & -1.526 & 0.0018 & 0.0095 \\
\hline & mmu-miR-382-3p & -2.192 & 0.0019 & 0.0096 \\
\hline & mmu-miR-193b-3p & -1.554 & 0.0020 & 0.0102 \\
\hline & mmu-miR-429-3p & -1.738 & 0.0021 & 0.0102 \\
\hline & mmu-miR-181b-5p & -1.485 & 0.0023 & 0.0109 \\
\hline & mmu-miR-1b-5p & -1.752 & 0.0024 & 0.0110 \\
\hline & mmu-miR-1a-3p & -1.751 & 0.0024 & 0.0111 \\
\hline & mmu-miR-450a-5p & -1.536 & 0.0025 & 0.0112 \\
\hline & mmu-miR-423-3p & -1.296 & 0.0034 & 0.0148 \\
\hline & mmu-miR-328-3p & -1.452 & 0.0035 & 0.0148 \\
\hline & mmu-miR-532-5p & -1.316 & 0.0048 & 0.0200 \\
\hline & mmu-miR-148a-5p & -1.521 & 0.0055 & 0.0212 \\
\hline & mmu-miR-499-5p & -1.473 & 0.0055 & 0.0212 \\
\hline & novel_115 & -2.137 & 0.0059 & 0.0223 \\
\hline & mmu-miR-425-5p & -1.490 & 0.0080 & 0.0283 \\
\hline & mmu-miR-299a-3p & -1.481 & 0.0091 & 0.0319 \\
\hline & mmu-miR-200a-3p & -1.500 & 0.0099 & 0.0342 \\
\hline & mmu-miR-30b-5p & -1.305 & 0.0104 & 0.0350 \\
\hline & mmu-miR-30e-5p & -1.486 & 0.0108 & 0.0356 \\
\hline & mmu-miR-200a-5p & -1.410 & 0.0110 & 0.0358 \\
\hline & mmu-miR-26a-5p & -1.260 & 0.0113 & 0.0363 \\
\hline & mmu-miR-99a-5p & -1.379 & 0.0125 & 0.0396 \\
\hline & mmu-miR-409-5p & -1.705 & 0.0127 & 0.0398 \\
\hline & mmu-miR-92a-3p & -1.350 & 0.0132 & 0.0409 \\
\hline & mmu-miR-129-1-3p & -1.769 & 0.0146 & 0.0446 \\
\hline & mmu-miR-148b-3p & -1.277 & 0.0167 & 0.0484 \\
\hline
\end{tabular}




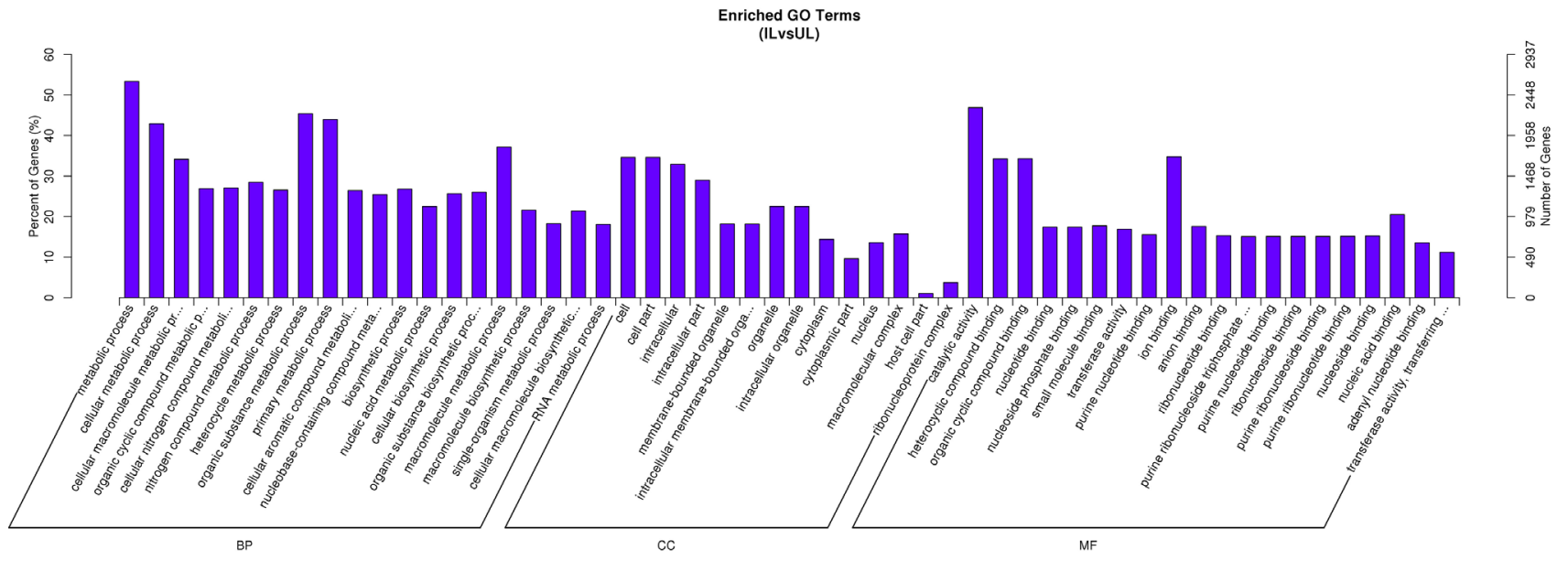

Figure 3: GO enrichment analysis of predicted gene targets of differentially expressed miRNAs. Abbreviations: IL vs UL: infected liver vs uninfected liver, BP: biological process, MF: molecular function, CC: cellular component.

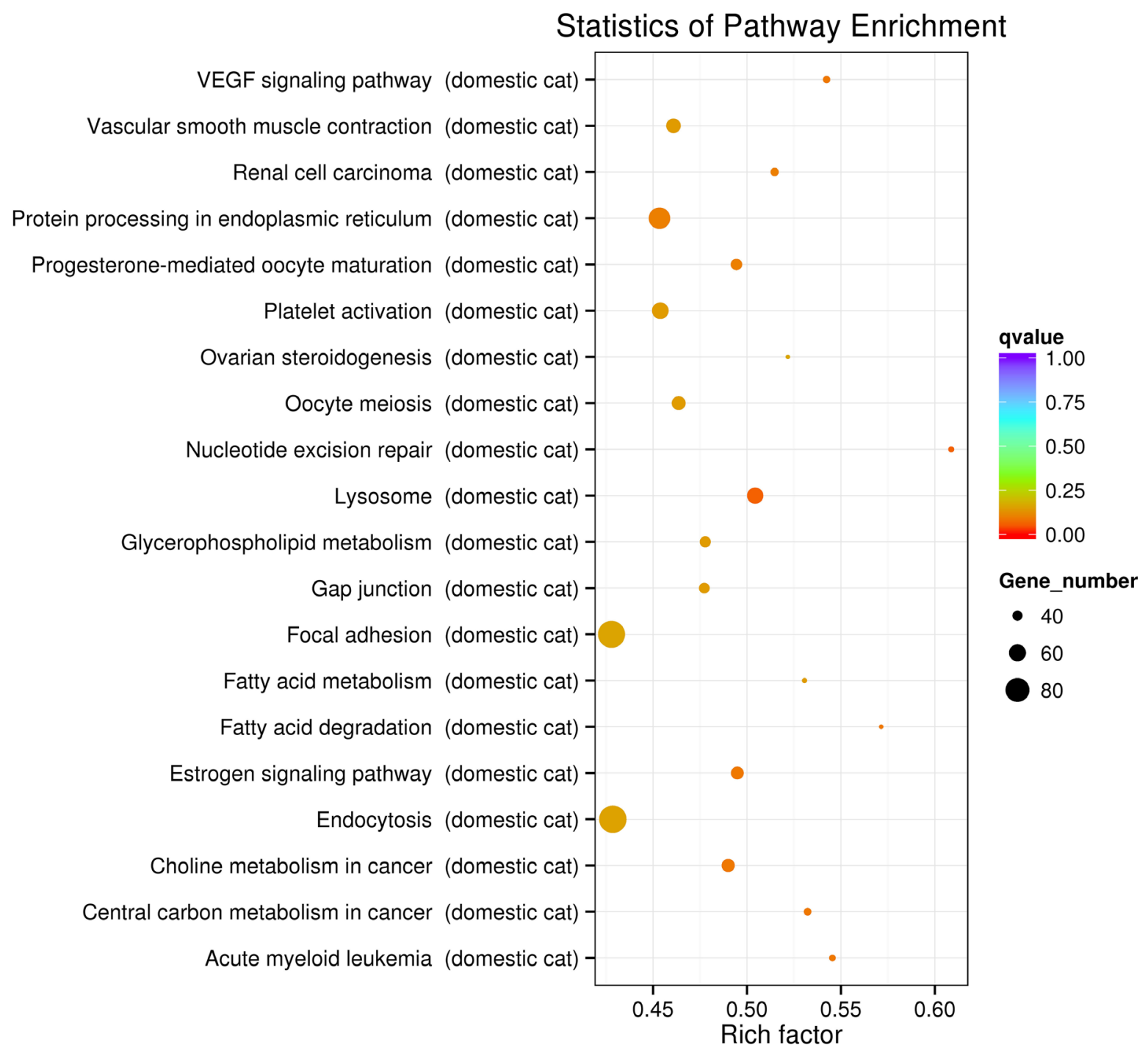

Figure 4: The top 20 enriched pathways of the predicted host targets of the differentially expressed miRNAs. 
modulate crucial host cellular targets needed for $T$. gondii replication, miRNAs may hold promise as biomarkers for prediction of disease progression. Finally, miRNA pathways that are stimulated during infection may offer potential targets for therapeutic control of toxoplasmosis.

\section{MATERIALS AND METHODS}

\section{Ethics statement}

This study was performed in strict accordance with the recommendations set forth in the Animal Ethics Procedures and Guidelines of the People's Republic of China. All animal experiments were reviewed and approved by the Animal Ethics Committee of Lanzhou Veterinary Research Institute, Chinese Academy of Agricultural Sciences (Approval No. LVRIAEC2014-009). Liver tissue collection was performed as a terminal procedure under isoflurane anesthesia and all efforts were made to minimize suffering.

\section{Animals, parasite infection and sample collection}

Twelve, 3 month-old, domestic cats (Felis catus) of the Chinese Li Hua breed were purchased from a local breeder and were housed in a controlled environment. The cats belonged to two litters, six cats per litter. These 12 cats were randomly allocated to four groups (two infected and two control) with three cats per group. Before the experiment, all cats were confirmed to be free from $T$. gondii using the modified agglutination test and free of major viral infections (e.g. feline calicivirus and coronavirus, feline immunodeficiency virus, feline leukemia virus, and feline parvovirus) based on serological examination. Cats were maintained on commercial cat diets (Royal Canine Inc., St. Charles, MO, USA) and water ad libitum during the 3 weeks prior to experimentation in order to allow cats to acclimate and to minimize any potential dietary influence on the study results. During the experiment cats were individually fed once daily based on daily energy requirements and water was available ad libitum.

Toxoplasma gondii strain used in this study was the PRU strain (Genotype II), which is maintained in our laboratory by passage through Kunming mice as described previously [49]. T. gondii type II was used in this study because it seems to be the predominant genotype circulating in cats [50-52]. Also, the PRU strain is able to produce brain tissue cysts in mouse and oocysts in the gut of cats and is thus a suitable candidate for a standardized challenge model in cats. The number of $T$. gondii cysts was determined using an optical microscope and was adjusted to 100 cysts $\mathrm{mL}^{-1}$ in phosphate buffered saline (PBS), $\mathrm{pH}$ 7.4. Each cat was infected by intragrastric inoculation with 100 cysts in $1 \mathrm{~mL}$ PBS. Control cats were sham-infected with PBS only. Livers were harvested 7 days post infection (7 dpi) in order to allow sufficient time for the infection to be established in the liver [1]. Collected livers were rinsed extensively in saline, flash frozen in liquid nitrogen, and stored at $-80^{\circ} \mathrm{C}$ until processing.

\section{Detection of infection in the liver}

Genomic DNA was extracted from the collected liver samples using TIANamp Genomic DNA kit according to the manufacturer's recommendations (TianGen ${ }^{\mathrm{TM}}$, Beijing, China). Then, a semi-nested PCR targeting $T$. gondii $B 1$ gene was performed to detect $T$. gondii infection [53]. DNA samples giving positive $B 1$ amplification were genotyped using PCR-restriction fragment length polymorphism analysis as described previously [54].

\section{RNA extraction and qualification}

Total RNA was prepared individually from the cryopreserved liver tissues of the cats using TRIzol Reagent according to the manufacturer's instructions (Invitrogen Co. Ltd). RNA degradation and contamination was checked on $1 \%$ agarose gels. RNA purity was evaluated using the NanoPhotometer ${ }^{\circledR}$ spectrophotometer (IMPLEN, CA, USA). RNA concentration was determined using Qubit ${ }^{\circledR}$ RNA Assay Kit in Qubit ${ }^{\circledR}$ 2.0 Flurometer (Life Technologies, CA, USA). RNA integrity was assessed using the RNA Nano 6000 Assay Kit of the Agilent Bioanalyzer 2100 system (Agilent Technologies, CA, USA).

\section{RNA sequencing library preparation and transcriptomic analysis}

RNA samples from $T$. gondii-infected and noninfected livers collected at $7 \mathrm{dpi}$ were sent to Beijing Novogene Bioinformatics Institute for Illumina sequencing. To analyze miRNAs by sequencing, a total of $3 \mu \mathrm{g}$ RNAs of three pooled samples from each group were used for the construction of four small RNA (sRNA) libraries, which were subjected to sequencing on a Hi-seq 2500 platform. Raw data (raw reads) of fastq format were firstly processed through custom perl and python scripts. In this step, clean reads were obtained by removing reads containing ploy-N, with 5 ' adapter contaminants, without 3 ' adapter or the insert tag, containing ploy A or T, or G or $\mathrm{C}$ and low quality reads from raw data. At the same time, Q20, Q30 and GC-content of the raw data were calculated. Then, we chose a certain range of length from clean reads to do all downstream analyses. Next, the small RNA tags were mapped to the feline reference genome sequence using Bowtie software [55]. The following parameters were used: -k [valid alignments per read], 1; -m [number of possible alignments], 10; -1 [seed length], 25; --best [optimal alignments]).

Mapped small RNA tags were used to look for known miRNA. miRBase20.0 was used as reference, and modified software mirdeep2 [56] and srna-tools-cli 
were used to obtain the potential miRNA and draw the secondary structures. Custom scripts were used to obtain the miRNA counts and base bias on the first position of identified miRNA with certain length and on each position of all identified miRNA, respectively. To remove tags originating from protein-coding genes, repeat sequences, rRNA, tRNA, snRNA, and snoRNA, small RNA tags were mapped to RepeatMasker, Rfam database or those types of data from the specified species itself.

The available software miREvo [57] and mirdeep2 [56] were integrated to predict novel miRNA through exploring the secondary structure, the dicer cleavage site and the minimum free energy of the small RNA tags unannotated in the former steps. miRNA expression levels were estimated with TPM (transcript per million) units [58]. Differential expression analysis of infected versus control groups was performed using the DESeq $\mathrm{R}$ package (1.8.3). The $P$-values was adjusted using the Benjamini \& Hochberg method. Corrected $P$-value of 0.05 was set as the threshold for significantly differential expression by default.

Predicting the target gene of miRNA was performed by psRobot_tar in miRanda [59]. Gene Ontology (GO) enrichment analysis was used to categorize the target genes of the differentially expressed miRNAs. GOseq based Wallenius non-central hyper-geometric distribution [60], which can adjust for gene length bias, was implemented for GO enrichment analysis. The enrichment of target genes in KEGG pathways were tested by the software KOBAS [61].

\section{Validation of miRNA expression}

The stem-loop quantitative reverse transcription PCR was used to validate the results of miRNA expression analysis as described previously [21, 62]. Stem-loop RTPCR was performed on ABI PRISM ${ }^{\circledR} 7500$ Sequence Detection System using SYBR Green qPCRSuperMix according to the manufacturer's protocol (Invitrogen). All qRT-PCR reactions were performed in three replicates. Gene expressions were calculated by $2^{-\Delta \Delta \mathrm{CT}}$ relative expression method as previously described [63]. snRNA U6 was used as normalization control in qRT-PCR.

\section{CONFLICTS OF INTEREST}

The authors declare that they have no competing interest.

\section{GRANT SUPPORT}

Project support was kindly provided by the National Natural Science Foundation of China (Grant No. 31230073) and the Fundamental Research Funds of Chinese Academy of Agricultural Sciences (Grant No. Y2016JC05).

\section{REFERENCES}

1. Dubey JP. Toxoplasmosis of animals and humans. Second edition. Boca Raton, Florida: CRC Press; 2010:313.

2. Montoya JG, Liesenfeld O. Toxoplasmosis. Lancet. 2004; 363:1965-1976.

3. Elsheikha HM. Congenital toxoplasmosis: priorities for further health promotion action. Public Health. 2008; 122:335-353.

4. Dubey JP, A. Zajac SA, Osofsky, Tobias L. Acute primary toxoplasmic hepatitis in an adult cat shedding Toxoplasma gondii oocysts. J Am Vet Med Assoc. 1990; 197:1616-1618.

5. Anfray P, Bonetti C, Fabbrini F, Magnino S, Mancianti F, Abramo F. Feline cutaneous toxoplasmosis: a case report. Vet Dermatol. 2005; 16:131-136.

6. Nagel SS, Williams JH, Schoeman JP. Fatal disseminated toxoplasmosis in an immunocompetent cat. J S Afr Vet Assoc. 2013; 84:E1-E6.

7. De Tommasi AS, Morini M, Turba ME, Otranto D, Bettini G. Hyperplastic cholangitis in a naturally Toxoplasma gondii-infected cat. Vet Q. 2014; 34:229-231.

8. Hill D, Dubey JP. Toxoplasma gondii: transmission, diagnosis and prevention. Clin Microbiol Infect. 2002; 8:634-640.

9. Spycher A, Geigy C, Howard J, Posthaus H, Gendron K, Gottstein B, Debache K, Herrmann DC, Schares G, Frey CF. Isolation and genotyping of Toxoplasma gondii causing fatal systemic toxoplasmosis in an immunocompetent 10-year-old cat. J Vet Diagn Invest. 2011; 23:104-108.

10. Foster SF, Charles JA, Canfield PJ, Beatty JA, Martin P. Reactivated toxoplasmosis in a FIV-positive cat. Aus Vet Pract. 1998; 28:159-163.

11. Dubey JP, Prowell M. Ante-mortem diagnosis, diarrhea, oocyst shedding, treatment, isolation, and genetic typing of Toxoplasma gondii associated with clinical toxoplasmosis in a naturally infected cat. J Parasitol. 2013; 99:158-160.

12. Atmaca HT, Dincel GC, Macun HC, Terzi OS, Uzunalioglu T, Kalender H, Kul O. A rare case of feline congenital Toxoplasma gondii infection: fatal outcome of systemic toxoplasmosis for the mother and its kitten. Berl Munch Tierarztl Wochenschr. 2013; 126:216-219.

13. He JJ, Ma J, Elsheikha HM, Song HQ, Zhou DH, Zhu XQ. Proteomic profiling of mouse liver following acute Toxoplasma gondii infection. PLoS One. 2016; 11:e0152022.

14. He JJ, Ma J, Elsheikha HM, Song HQ, Huang SY, Zhu XQ. Transcriptomic analysis of mouse liver reveals a potential hepato-enteric pathogenic mechanism in acute Toxoplasma gondii infection. Parasit Vectors. 2016; 9:427.

15. Bartel DP. MicroRNAs: genomics, biogenesis, mechanism, and function. Cell. 2004; 116:281-297.

16. Carrington JC, Ambros V. Role of microRNAs in plant and animal development. Science. 2003; 301:336-338. 
17. Ha M, Kim VN. Regulation of microRNA biogenesis. Nat Rev Mol Cell Biol. 2014; 15:509-524.

18. Krol J, Loedige I, Filipowicz W. The widespread regulation of microRNA biogenesis, functions and decay. Nat Rev Genet. 2010; 33:597-610.

19. Benhamou Y, Di Martino V, Bochet M, Colombet G, Thibault V, Liou A, Katlama C, Poynard T, MultivirC Group. Factors affecting liver fibrosis in human immunodeficiency virus-and hepatitis $\mathrm{C}$ virus-coinfected patients: impact of protease inhibitor therapy. Hepatology. 2001; 34:283-287.

20. Judice CC, Bourgard C, Kayano ACAV, Albrecht L, Costa FTM. MicroRNAs in the host-apicomplexan parasites interactions: a review of immunopathological aspects. Front Cell Infect Microbiol. 2016; 6:5.

21. He JJ, Ma J, Wang JL, Xu MJ, Zhu XQ. Analysis of miRNA expression profiling in mouse spleen affected by acute Toxoplasma gondii infection. Infect Genet Evol. 2016; 37:137-142.

22. Shapira S, Speirs K, Gerstein A, Caamano J, Hunter CA. Suppression of NF-kappaB activation by infection with Toxoplasma gondii. J Infect Dis. 2002; 185:S66-S72.

23. Zeiner GM, Norman KL, Thomson JM, Hammond SM, Boothroyd JC. Toxoplasma gondii infection specifically increases the levels of key host microRNAs. PLoS One. 2010; 5:e8742.

24. Xiao C, Rajewsky K. MicroRNA control in the immune system: basic principles. Cell. 2009; 136:26-36.

25. Lopes-Ramos CM, Habr-Gama A, Quevedo Bde S, Felício NM, Bettoni F, Koyama FC, Asprino PF, Galante PA, Gama-Rodrigues J, Camargo AA, Perez RO, Parmigiani RB. Overexpression of miR-21-5p as a predictive marker for complete tumor regression to neoadjuvant chemoradiotherapy in rectal cancer patients. BMC Med Genomics. 2014; 7:68.

26. Tian F, Li R, Chen Z, Shen Y, Lu J, Xie X, Ge Q. Differentially expressed miRNAs in tumor, adjacent, and normal tissues of lung adenocarcinoma. Biomed Res Int. 2016; 2016:1428271.

27. Park SK, Park YS, Ahn JY, Do EJ, Kim D, Kim JE, Jung K, Byeon JS, Ye BD, Yang DH, Park SH, Hwang SW, Jung $\mathrm{HY}$, et al. MiR 21-5p as a predictor of recurrence in young gastric cancer patients. J Gastroenterol Hepatol. 2016; 31:1429-1435.

28. Zeiner GM, Norman KL, Thomson JM, Hammond SM, Boothroyd JC. Toxoplasma gondii infection specifically increases the levels of key host microRNAs. PLoS One. 2010; 5:e8742.

29. Ernst A, Campos B, Meier J, Devens F, Liesenberg F, Wolter M, Reifenberger G, Herold-Mende C, Lichter P, Radlwimmer B. De-repression of CTGF via the miR-17-92 cluster upon differentiation of human glioblastoma spheroid cultures. Oncogene. 2010; 29:3411-3422.
30. Braun L, Cannella D, Ortet P, Barakat M, Sautel CF, Kieffer S, Garin J, Bastien O, Voinnet O, Hakimi M-A. A complex small RNA repertoire is generated by a plant/fungal-like machinery and effected by a metazoan-like argonaute in the single-cell human parasite Toxoplasma gondii. PLoS Pathog. 2010; 6:e1000920.

31. Grundhoff A, Sullivan CS. Virus-encoded microRNAs. Virology. 2011; 411:325-343.

32. Zhou R, Hu G, Liu J, Gong AY, Drescher KM, Chen XM. NF-kappaB p65-dependent transactivation of miRNA genes following Cryptosporidium parvum infection stimulates epithelial cell immune responses. PLoS Pathog. 2009; 5:e1000681.

33. Johnnidis JB, Harris MH, Wheeler RT, Stehling-Sun S, Lam MH, Kirak O, Brummelkamp TR, Fleming MD, Camargo FD. Regulation of progenitor cell proliferation and granulocyte function by microRNA-223. Nature. 2008; 451:1125-1129.

34. Xiao C, Rajewsky K. MicroRNA control in the immune system: basic principles. Cell. 2009; 136:26-36.

35. Hossain A, Kuo MT, Saunders GF. Mir-17-5p regulates breast cancer cell proliferation by inhibiting translation of AIB1 mRNA. Mol Cell Biol. 2006; 26:8191-8201.

36. He L, Thomson JM, Hemann MT, Hernando-Monge E, Mu D, Goodson S, Powers S, Cordon-Cardo C, Lowe SW, Hannon GJ, Hammond SM. A microRNA polycistron as a potential human oncogene. Nature. 2005; 435:828-833.

37. Ferreira RB, Ferreira R, Albuquerque DM, Costa FF, Franco-Penteado CF. miRNA-146a, miRNA-203a, and miRNA-223 modulate inflammatory response in LPS- acute lung injury in Sickle Cell transgenic mice. Blood. 2015; 126:3390.

38. Johnnidis JB, Harris MH, Wheeler RT, Stehling-Sun S, Lam MH, Kirak O, Brummelkamp TR, Fleming MD, Camargo FD. Regulation of progenitor cell proliferation and granulocyte function by microRNA-223. Nature. 2008; 451:1125-1129.

39. Xu J, Yao Q, Hou Y, Xu M, Liu S, Yang L, Zhang L, Xu H. MiR-223/Ect2/p21 signaling regulates osteosarcoma cell cycle progression and proliferation. Biomed Pharmacother. 2013; 67:381-386.

40. Faraoni I, Antonetti FR, Cardone J, Bonmassar E. miR155 gene: a typical multifunctional microRNA. Biochim Biophys Acta Mol Basis Dis. 2009; 1792:497-505.

41. Vigorito E, Kohlhaas S, Lu D. Leyland R. miR-155: an ancient regulator of the immune system. Immunol Rev. 2013; 253:146-157.

42. Taganov KD, Boldin MP, Chang KJ, Baltimore D. NF-kappaB-dependent induction of microRNA miR146, an inhibitor targeted to signaling proteins of innate immune responses. Proc Natl Acad Sci USA. 2006; 103:12481-12486. 
43. Saba R, Sorensen DL, Booth SA. MicroRNA-146a: a dominant, negative regulator of the innate immune response. Front Immunol. 2014; 5:578.

44. Ferretti C, La Cava A. miR-126, a new modulator of innate immunity. Cell Mol Immunol. 2014;11:215-217.

45. Song L, Lin C, Gong H, Wang C, Liu L, Wu J, Tao S, Hu B, Cheng SY, Li M, Li J. miR-486 sustains NF- $\kappa$ B activity by disrupting multiple NF-кB-negative feedback loops. Cell Res. 2013; 23:274-289.

46. Chen XM, Splinter PL, O'Hara SP, LaRusso NF. A cellular micro-RNA, let-7i, regulates Toll-like receptor 4 expression and contributes to cholangiocyte immune responses against Cryptosporidium parvum infection. J Biol Chem. 2007; 282:28929-28938.

47. Cannella D, Brenier-Pinchart MP, Braun L, van Rooyen JM, Bougdour A, Bastien O, Behnke MS, Curt RL, Curt A, Saeij JP, Sibley LD, Pelloux H, Hakimi MA. MiR-146a and miR-155 delineate a microRNA fingerprint associated with Toxoplasma persistence in the host brain. Cell Rep. 2014; 6:928-937.

48. Jia B, Chang Z, Wei X, Lu H, Yin J, Jiang N1, Chen Q. Plasma microRNAs are promising novel biomarkers for the early detection of Toxoplasma gondii infection. Parasit Vectors. 2014; 7:433.

49. Yan HK, Yuan ZG, Song HQ, Petersen E, Zhou Y, Ren D, Zhou DH, Li HX, Lin RQ, Yang GL, Zhu XQ. Vaccination with a DNA vaccine coding for perforin-like protein 1 and MIC6 induces significant protective immunity against Toxoplasma gondii. Clin Vaccine Immunol. 2012; 19:684-689.

50. Herrmann DC, Pantchev N, Vrhovec MG, Barutzki D, Wilking H, Fröhlich A, Lüder CG, Conraths FJ, Schares G. Atypical Toxoplasma gondii genotypes identified in oocysts shed by cats in Germany. Int J Parasitol. 2010; 40:285-292.

51. Maksimov P, Zerweck J, Dubey JP, Pantchev N, Frey CF, Maksimov A, Reimer U, Schutkowski M, Hosseininejad M, Ziller M, Conraths FJ, Schares G. Serotyping of Toxoplasma gondii in cats (Felis domesticus) reveals predominance of Type II infections in Germany. PLoS One. 2013; 8: e80213.

52. Brennan A, Donahoe SL, Beatty JA, Belov K, Lindsay S, Briscoe KA, Šlapeta J, Barrs VR. Comparison of genotypes of Toxoplasma gondii in domestic cats from Australia with latent infection or clinical toxoplasmosis. Vet Parasitol. 2016; 228:13-16.
53. Cong W, Qin SY, Meng QF, Zou FC, Qian AD, Zhu XQ. Molecular detection and genetic characterization of Toxoplasma gondii infection in sika deer (Cervus nippon) in China. Infect Genet Evol. 2016; 39:9-11.

54. Cong W, Liu GH, Meng QF, Dong W, Qin SY, Zhang FK, Zhang XY, Wang XY, Qian AD, Zhu XQ. Toxoplasma gondii infection in cancer patients: Prevalence, risk factors, genotypes and association with clinical diagnosis. Cancer Lett. 2015; 359:307-313.

55. Langmead B, Trapnell C, Pop M, Salzberg SL. Ultrafast and memory-efficient alignment of short DNA sequences to the human genome. Genome Biol. 2009; 10:R25.

56. Friedlander MR, Mackowiak SD, Li N, Chen W, Rajewsky N. miRDeep2 accurately identifies known and hundreds of novel microRNA genes in seven animal clades. Nucleic Acids Res. 2011; 40:37-52.

57. Wen M, Shen Y, Shi S, Tang T. miREvo: An integrative microRNA evolutionary analysis platform for nextgeneration sequencing experiments. BMC Bioinformatics. 2010; 13:140.

58. Zhou L, Chen J, Li Z, Li X, Hu X, Huang Y, Zhao X, Liang C, Wang Y, Sun L, Shi M, Xu X, Shen F, et al. Integrated profiling of microRNAs and mRNAs: microRNAs located on Xq27.3 associate with clear cell renal cell carcinoma. PLoS One. 2010; 5:e15224.

59. Enright AJ, John B, Gaul U, Tuschl T, Sander C, Marks DS. MicroRNA targets in Drosophila. Genome Biol. 2003; 5:R1.

60. Chen CM, Lu YL, Sio CP, Wu G, Tzou WS, Pai TW. Gene Ontology based housekeeping gene selection for RNA-seq normalization. Methods. 2014; 67:354-363.

61. Mao X, Cai T, Olyarchuk JG, Wei L. Automated genome annotation and pathway identification using the KEGG orthology (KO) as a controlled vocabulary. Bioinformatics. 2005; $21: 3787-3793$.

62. Chen C, Ridzon DA, Broomer AJ, Zhou Z, Lee DH, Nguyen JT, Barbisin M, Xu NL, Mahuvakar VR, Andersen MR. Real-time quantification of microRNAs by stem-loop RT-PCR. Nucleic Acids Res. 2005; 33:e179.

63. Livak KJ, Schmittgen TD. Analysis of relative gene expression data using realtime quantitative PCR and the ${ }^{-{ }^{\Delta}} \mathrm{CT}$ method. Methods. 2001; 25:402-408. 